\title{
Development of truly portable track geometry recording trolley and accompanying new measurement principle
}

\author{
Y. Naganuma \& T. Yada \\ Technology Research and Development Department, \\ Central Japan Railway Company, Japan
}

\begin{abstract}
In 2015, a truly portable track geometry measuring trolley was successfully developed to enable real-time measurement after all track maintenance work. An experimental model named LRSX01 (development code) was realized that is small (disassembled total length of $1600 \mathrm{~mm}$ maximum) and lightweight $(9.5 \mathrm{~kg}$, which is about one-third that of track geometry measuring trolley LIGHTREC). Seven items may be measured in real-time; track gauge, cross level, $10 \mathrm{~m}$ alignment (right and left), $10 \mathrm{~m}$ longitudinal level (right and left), and twist. Measuring accuracy is high, and it far outperforms current track geometry measuring trolleys in terms of repeatability error. Mechanism of the LRSX01 is very simple and easy to maintain because there are no moving parts for measuring alignment and longitudinal level. These advantages, which are not found on any similar conventional product, were achieved with a brand-new track geometry measuring principle called "differential difference method" for the LRSX01. The LRSX01 product model will go on sale globally in April 2017.

Keywords: track geometry measuring trolley, differential difference method, MEMS gyroscope.
\end{abstract}

\section{Introduction}

As part of maintenance planning at JR-Central, track geometry is measured by a high-speed track recording car every 10 days on the Tokaido Shinkansen [1] and every month on conventional lines. To confirm the state of the track after 
maintenance work, a measuring system built into the maintenance machinery is frequently used after ballast renewal work and a track geometry measuring trolley known as LIGHTREC [2] is used after concrete sleeper renewal. However, even today, track that is maintained manually is still measured by means of a manual survey using a chord and scale. Many types of portable measuring equipment and trolleys, including LIGHTREC, have been developed and sold globally. Nevertheless, these are rarely used after manual work, because most equipment is too large and heavy to transport and remove from the track when a train passes. Moreover, it is too expensive to purchase the number of devices required for every maintenance site.

To provide a solution for such problems, in 2015, we once again launched development of a small, inexpensive and lightweight track geometry recording trolley that utilizes the latest sensors and data processing technology with the aim of introducing a device for measuring track geometry of all maintenance work.

\section{Conventional measurement principal}

\subsection{Second-order difference method}

Most conventional equipment and trolleys adopt the second-order difference method. Since this measures track geometry by detecting the relative displacement of three points, it has also been called the three-point method.

Figure 1(a) shows a typical setup for the measurement of longitudinal level or alignment using the second-order difference method. A stiff beam (about 1.0 $3.0 \mathrm{~m}$ ), which is the measuring standard, is set along a rail, and the relative distance between the center point of the beam and rail is measured using a displacement gauge. The measurement beam is required to have a sufficiently high rigidity, because even a slight deflection of the beam results in a large error in the measurement. This beam requirement has prevented the miniaturization as well as weight and cost reduction for portable measuring equipment.

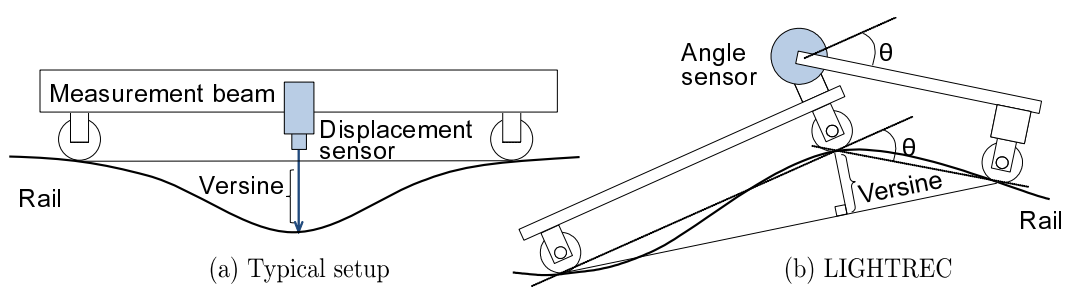

Figure 1: Example of setup for second-order difference measurement.

Figure 1(b) shows another setup for the second-order difference method, which has been devised for LIGHTREC. Utilizing angle measurement gives the following two advantages: 
(a) Beams can be folded to half the chord length for transportation.

(b) Because the force on each component is small, LIGHTREC is able to use slender beams, significantly reducing overall weight.

\subsection{First-order difference method}

A few types of track geometry measuring trolleys do not adopt the second-order difference method. In one type, a trolley detects the slope of the track (pitch of the trolley) using an inclinometer, which is equipped for standard measuring, and then the longitudinal level is obtained by integrating the slope over the distance [3]. This method measures the difference between two points of the track geometry, so it is called the first-order difference method or the two-point method. Since the firstorder difference method requires two measuring points, it is an attractive method for measuring track geometry. However, it is difficult to apply for alignment, because an expensive sensor such as an IMU (Inertial Measurement Unit) is required to detect the yaw angle.

\section{New measurement method for LRSX01}

\subsection{Two-point angle method}

For high accuracy profile measurements, a measuring method known as the twopoint angle method is frequently used [4]. Figure 2 shows a schematic of the principle behind this method. Two optical sensors, known as laser autocollimators, are mounted on a measurement stage to determine the angle, which is the first stage distance differential, to measure the shape according to the differential output (difference) between the surface of the object and the stage. A differential operation for the two sensors can be used to cancel errors due to pitching and bouncing motion of the stage.

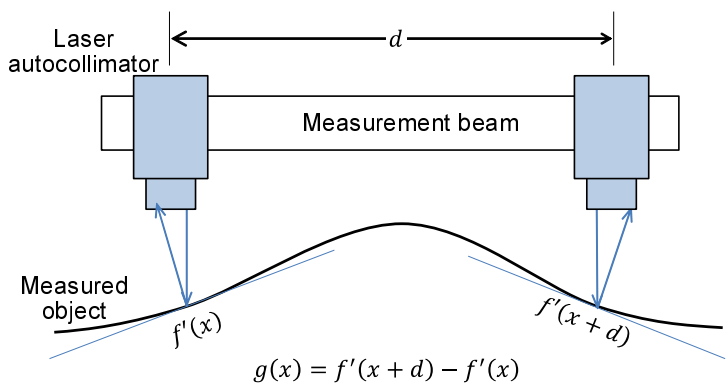

Figure 2: Principle of the two-point angle method (moving laser autocollimator).

Just as with the first-order difference method, the two-point angle method requires only two measurement points, so it presents a highly attractive method for 
measuring track geometry. However, because the laser autocollimator is affected by the reflection factor of the measurement object, it is difficult to apply this method to track geometry measurement because rails are not ideal surfaces for an autocollimator as reflected light is dampened due to grime and reflected fully at wheel contact points.

\subsection{Differential difference method}

The above two-point method was modified and adopted as the measuring principle for the LRSX01. If angular velocity, which is a time derivative, is converted into an inclination, which is a distance derivative, the two-point angle method is applicable to track geometry measurement using only an inexpensive MEMS gyroscope instead of expensive IMU or autocollimator. Based on this idea, a method which uses a gyroscope to obtain the same result as the two-point angle method is illustrated in Figure 3.

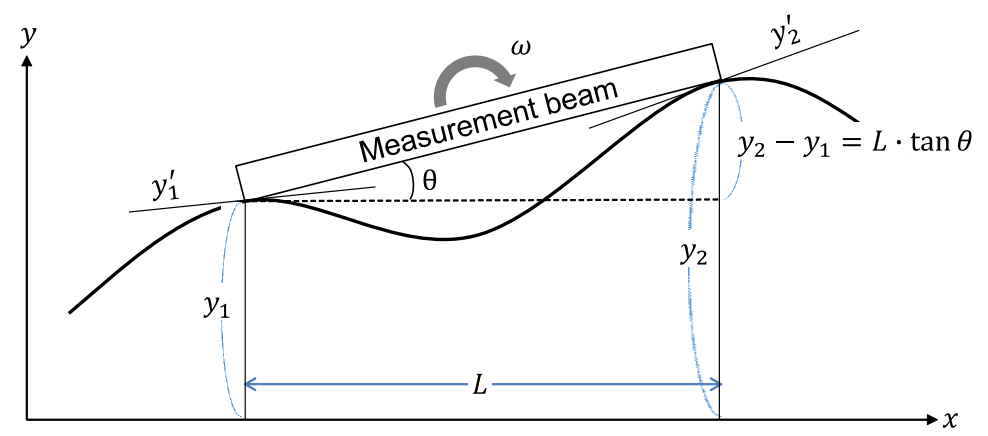

Figure 3: New track geometry measurement method using gyroscope.

A variable $g$ is defined as a difference of gradients at two points in Figure 3.

$$
g=y_{2}^{\prime}-y_{1}^{\prime}
$$

Equation (2) is obvious from the figure.

$$
y_{2}-y_{1}=L \cdot \tan \theta
$$

Then, differentiating both sides of the eqn (2) results in the following eqn (3).

$$
y_{2}^{\prime}-y_{1}^{\prime}=(L \cdot \tan \theta)^{\prime}=L \frac{1}{\cos ^{2} \theta} \cdot \frac{d \theta}{d x}=L \frac{1}{\cos ^{2} \theta} \cdot \frac{d \theta}{d t} \cdot \frac{d t}{d x}
$$

By putting $d \theta / d t=\omega$ (angler velocity), $d x / d t=v$ (measuring velocity) and introducing approximation of $\cos \theta \cong 1$ because $\theta$ is relatively small, the 
difference of gradient $g$ is expressed in the following simple equation.

$$
g \cong \frac{L \omega}{v}
$$

Equation (4) shows that track geometry, which does not depend on the measuring speed, is provided by following procedure.

(a) A gyroscope is put on a measurement beam of length $L$.

(b) $L$ is multiplied by output pitch angular velocity (or yaw angle velocity) from the gyroscope.

(c) The result is divided by the measured velocity $v$.

This method uses the gyroscope which is an inertial sensor, but does not necessitate the integral calculus, which is usually required. Therefore, it is unrelated to the drift and revolutionary in that it always stably measures track geometry.

This newly devised principle has the same measurement characteristics as the two-point angle method, but the sensor configuration is very different. Moreover, this method has not been utilized at all for track geometry measurement. Therefore, we named this new track measuring method the "differential difference method (DD-method)."

\subsection{Measurement characteristics of the DD-method}

The DD-method is expressed as eqn (5) when $L=2 l$.

$$
g(x)=(y(x+l)-y(x-l))^{\prime}
$$

The frequency response function $H(\omega)$ is expressed as eqn (6) using Fourier transform of eqn (5).

$$
H(\omega)=j \omega\left(e^{j \omega t}-e^{-j \omega t}\right)=-2 \omega \sin \omega l
$$

Gain and phase characteristics are expressed as shown in eqn (7).

$$
\begin{aligned}
& |H(\omega)|=2 \omega|\sin \omega l| \\
& \theta(\omega)=0
\end{aligned}
$$

The gain of the DD-method and existing methods are compared in Figure 4. Since its gain in a short wavelength range is relatively high, the DD-method may diagnose the state of rail roughness at the same time as it measures track geometry.

\section{Conversion to versine data}

As shown in eqn (1), the DD signal data is a first-order difference of distance differential, so it can be converted to versine, which is a second-order difference, by using a calculation for distance integration and first-order difference. In this 


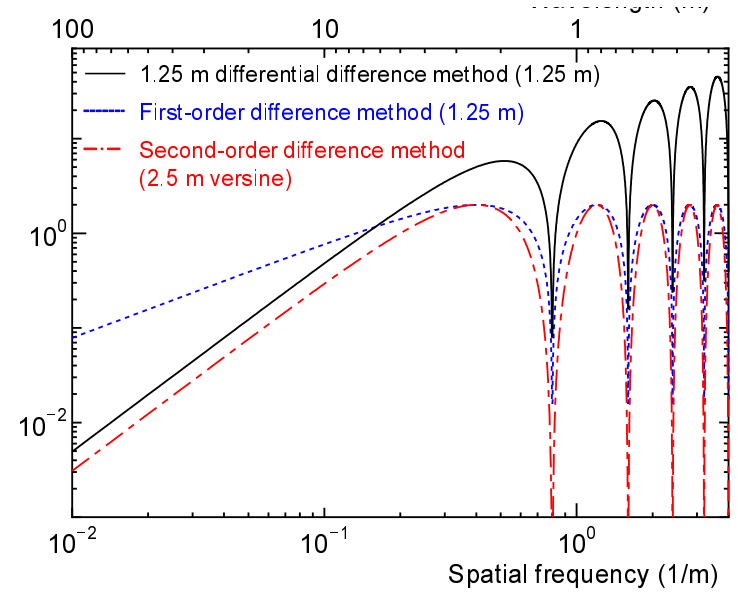

Figure 4: Gain characteristics of the DD-method.

section, the conversion procedure to versine is explained and a more effective method is described.

The $2.5 \mathrm{~m}$ versine method may be expressed by eqn (8):

$$
v(x)=y(x)-\frac{y(x+1.25)+y(x-1.25)}{2}
$$

where,

$y(\xi)$ : measured $2.5 \mathrm{~m}$ versine signal $x(\xi)$ : track profile

From eqn (8), a transfer function for a $2.5 \mathrm{~m}$ versine measurement on the z-plane yields eqn (9). In this equation, the sampling distance is $0.25 \mathrm{~m}$ with an output delay of $1.25 \mathrm{~m}$ to satisfy the principle of causality.

$$
H_{V S}(z)=-\frac{1}{2}+z^{-5}-\frac{1}{2} z^{-10}=-\frac{1}{2}\left(1-z^{-5}\right)^{2}
$$

Equation (9) shows that a characteristic of the $2.5 \mathrm{~m}$ versine consists of two difference filters and one multiplier [5]. Figure 5 shows a block diagram of the $2.5 \mathrm{~m}$ versine method.

And from eqn (5), transfer function of the DD-method is expressed as eqn (10). A block diagram of the DD-method is shown in Figure 6.

$$
H_{D D}(z)=\underbrace{\left(1-z^{-1}\right)}_{\text {differential difference }} \underbrace{\left(1-z^{-5}\right)}
$$




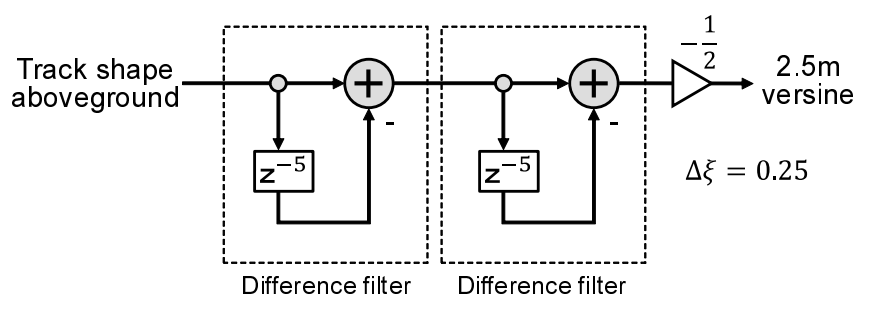

Figure 5: Block diagram of the $2.5 \mathrm{~m}$ versine method.

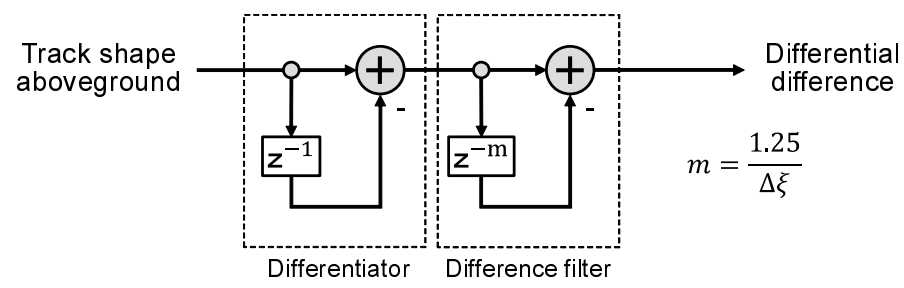

Figure 6: Block diagram of the $1.25 \mathrm{~m}$ DD-method.

From eqn (9) and (10), the conversion from DD-signal data to versine is shown in eqn (11).

$$
H_{V S}(z)=-\frac{1}{2} \underbrace{\left(1-z^{-5}\right)}_{\text {difference }} \underbrace{\left(\frac{1}{1-z^{-1}}\right)}_{\text {integral }} \cdot H_{D D}(z)
$$

Equation (11) shows that "after the difference and integration are executed for the DD-data, and coefficient -0.5 is multiplied, the product is versine." The block diagram is shown in Figure 7.

Furthermore, the product of difference $\left(1-z^{-5}\right)$ and integrator $\left(1 /\left(1-z^{-1}\right)\right)$ becomes a moving summation by polynomial division.

$$
\frac{1-z^{-5}}{1-z^{-1}}=1+z^{-1}+z^{-2}+z^{-3}+z^{-4}
$$

The above equation shows that the DD-signal data is completely converted to versine by the simple and stable calculus of moving summation. Figure 8 shows a block diagram of the versine conversion algorithm of moving summation.

As stated in this section, measurement by DD-method and conversion to versine are very simple and may be performed with minimal operation. The simple data processing contributes to cost reduction and electric power savings for commercialization of the product because the latest CPU is not necessary. 


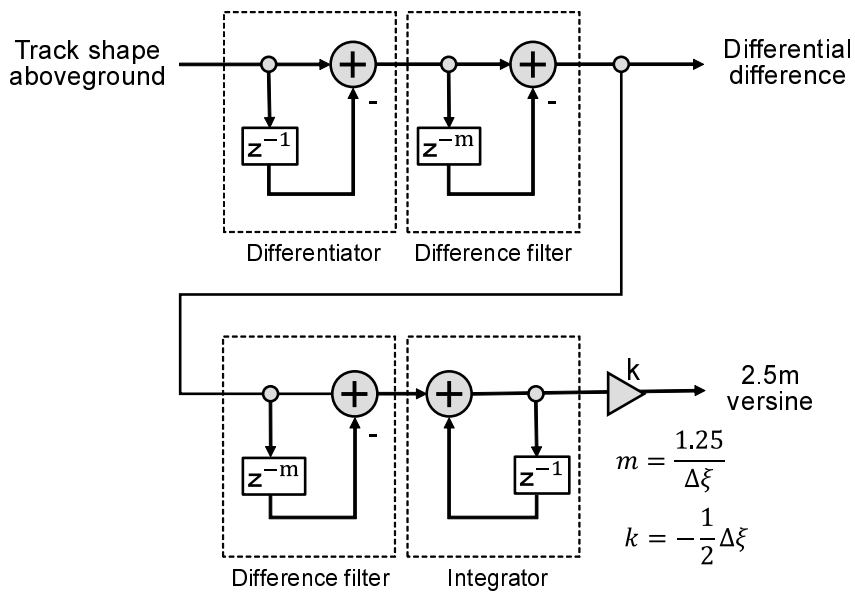

Figure 7: Conversion from a DD-signal data to versine signal by difference filter and integrator (1).

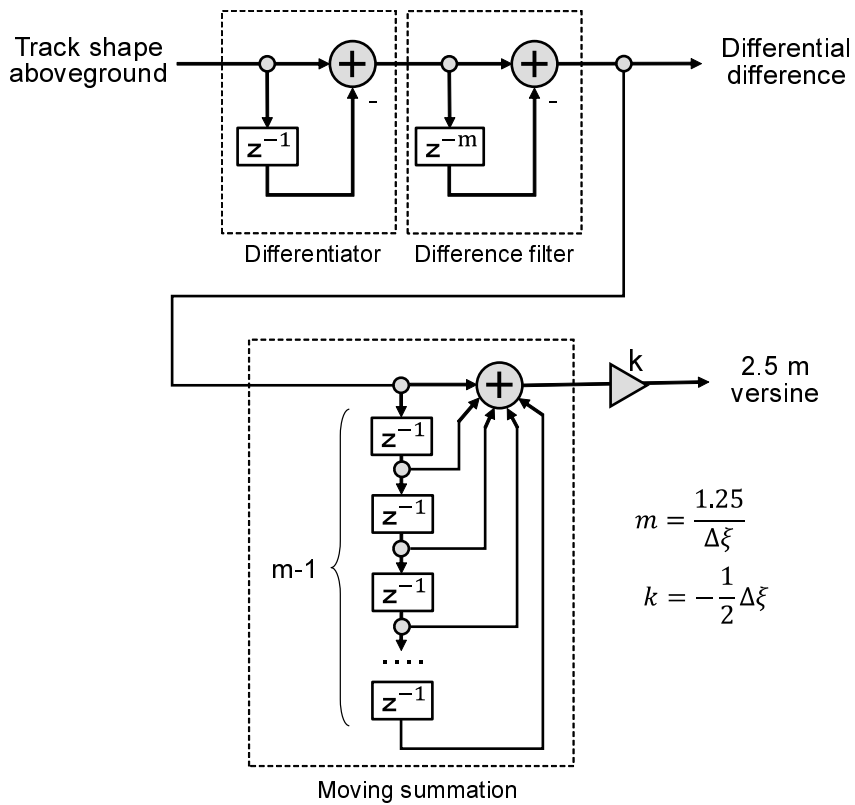

Figure 8: Conversion from a DD-signal data to versine signal by moving summation (2). 


\section{Experimental prototype LRSX01}

Figure 9 shows an experimental prototype which adopts the DD-method. It is called LRSX01 in development code. The weight of the body (excluding a pushpull bar and display unit) is a very light at $9.5 \mathrm{~kg}$. The measurement rail side requires only two wheels. Furthermore, there are no mechanical moving parts for measuring alignment and longitudinal level. All these features have been achieved by the adoption of the newly developed DD-method.

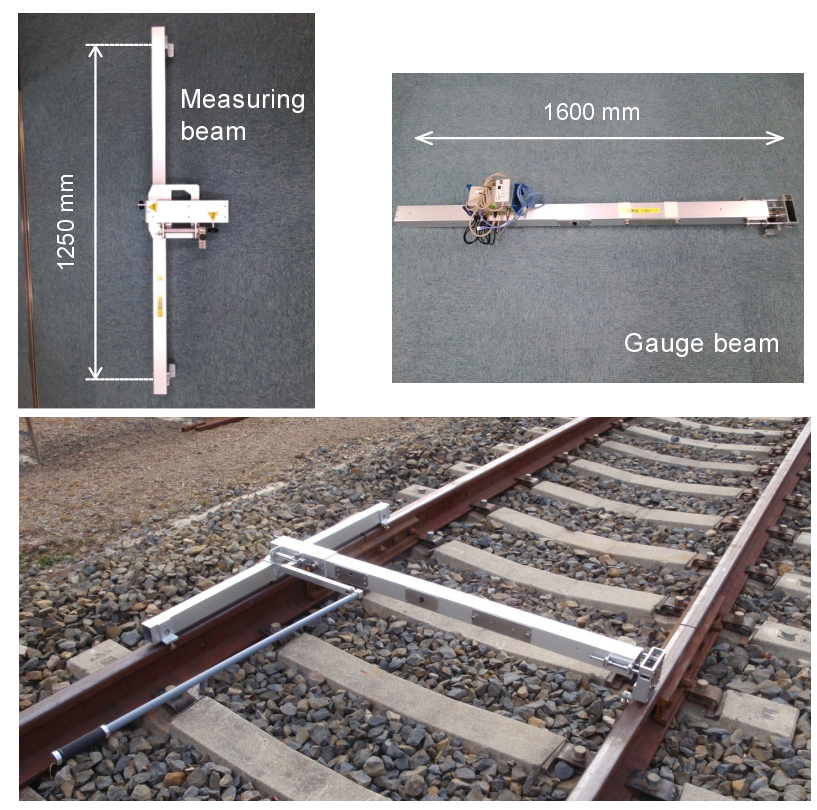

Figure 9: Experimental prototype model LRSX01.

The body is divided into a two-part structure with a "gauge beam" and "measurement beam." The assembly is completed by inserting the gauge beam to the measuring beam and tightening two dial screws. Assembly requires less than 20 seconds.

\section{Measurement accuracy and repeatability}

As is clear from eqn (4), the angular velocity signal is divided by measurement velocity $(v)$ to convert time-differential into distance-differential. Therefore, when the measuring speed is extremely low, there was concern about divergence in the calculation results. To ascertain this impact, repeatability tests were performed by modifying the measuring speed to 2,4 and $6 \mathrm{~km} / \mathrm{h}$ as well as stopping midway. The results for six tests for alignment are shown in Figure 10 together with the 
measuring speeds. As a result of examination, the impact of measuring speed was found to be almost nonexistent. This reason is that data for speeds close to zero are not input due to distance sampling.

The results of a repeatability test for longitudinal level are shown in Figure 11. The repeatability of both alignment and longitudinal level is very high in a wide wavelength range $(2.5,10,20,40 \mathrm{~m}$ versine), showing that lightweight and highaccuracy track geometry measuring trolley has become a reality.

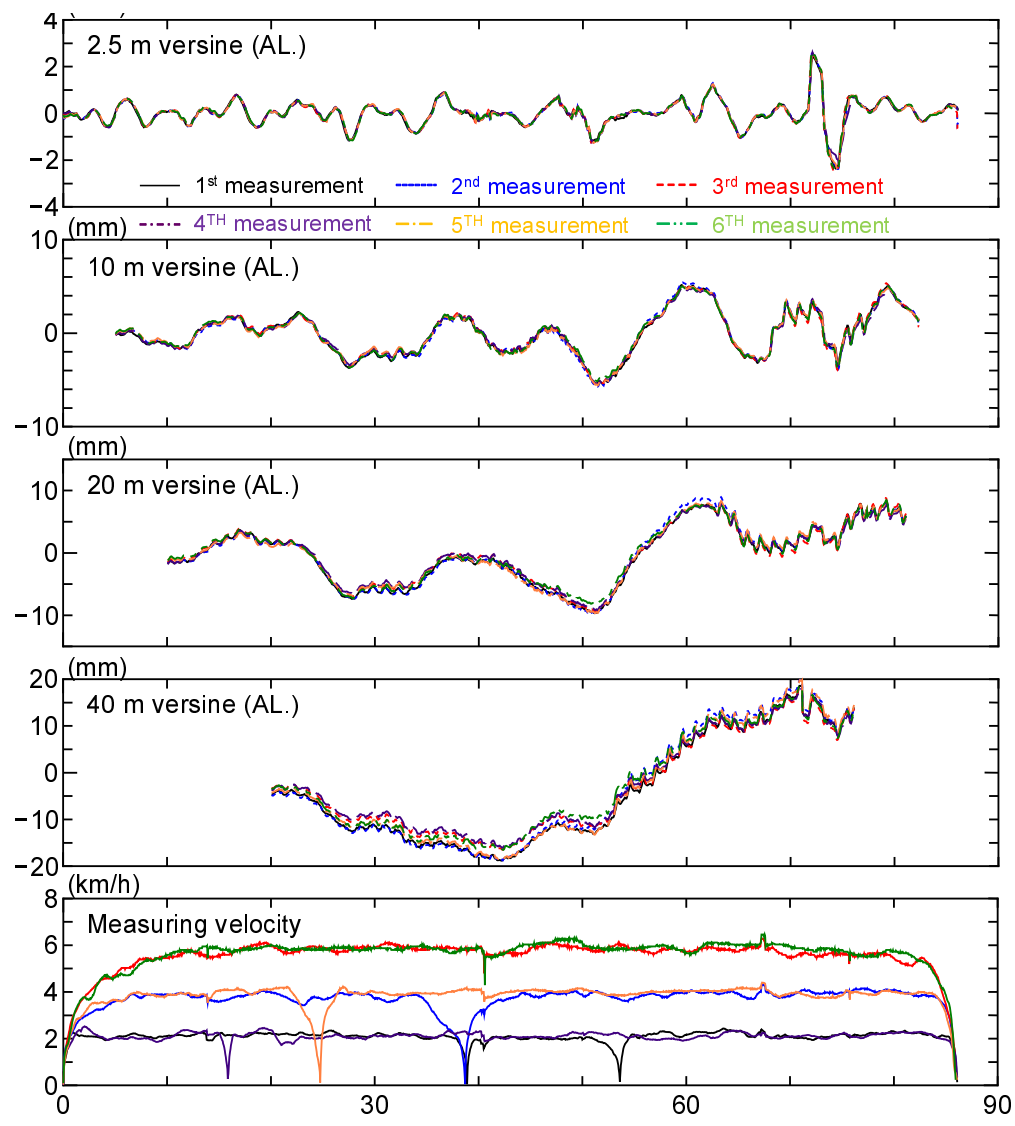

Figure 10: Results of repeatability tests of LRSX01 (alignment).

\section{Conversion to D1 track geometry}

This section discusses conformance with the European standard series EN 13848. Because EN13848-4 requires the track geometry index known as D1 rather than versine, conversion to the D1 is indispensable for the development to a worldwide 

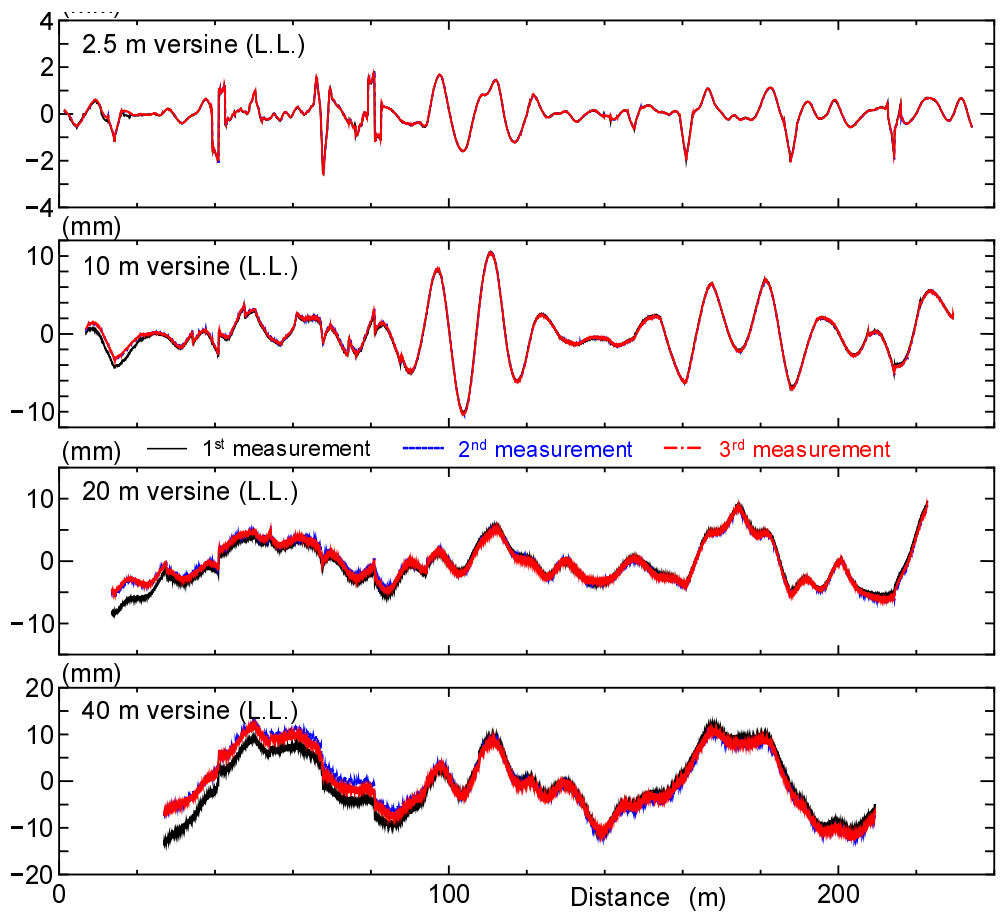

Figure 11: Results of repeatability tests of LRSX01 (longitudinal level).

market. D1 track geometry is a band-limited true track profile and the waveband ranges from $3.0 \mathrm{~m}$ to $25 \mathrm{~m}$. There are two methods for converting to D1: a method calculating from $2.5 \mathrm{~m}$ versine and a method calculating directly from DD-signal data. This paper addresses a re-coloring filter for DD-signal. Conditions of the designed re-coloring filter are shown in Table 1.

In Figure 12, (a) indicates the impulse response function (filter coefficient), (b) indicates the frequency response function of re-coloring filter with DD measurement and the comprehensive frequency response function given as the result of re-coloring.

Results of the repeatability tests of D1 signals measured from LRSX01 are shown in Figure 13. In the case of an FIR filter, the output signal has a transient response, which decreases calculation precision, in the beginning and end, and it is $1 / 2$ length of the impulse response. Therefore, the transient response length of the designed re-coloring filter is $75 \mathrm{~m}$. The range of the transient responses are shown in Figure 13.

The repeatability error of D1 track geometry was calculated as follows. 
Table 1: Design conditions of re-coloring filter for DD measurement data.

\begin{tabular}{cc}
\hline Sampling spacing & $0.05 \mathrm{~m}$ \\
Number of impulse response(Number of the taps) & 3001 \\
Pass band & $3 \mathrm{~m} \sim 25 \mathrm{~m}$ \\
Transition band (short wavelength) & $1.25 \mathrm{~m} \sim 3 \mathrm{~m}$ \\
Transition band (long wavelength) & $25 \mathrm{~m} \sim 50 \mathrm{~m}$ \\
Gain of the stop band & 0.0001 \\
\hline
\end{tabular}

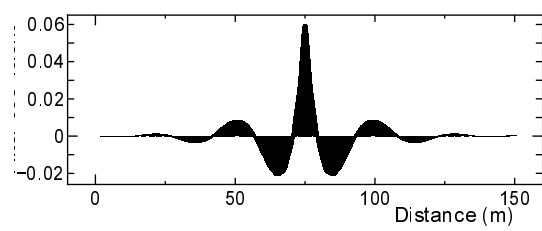

(a) Impulse response functions

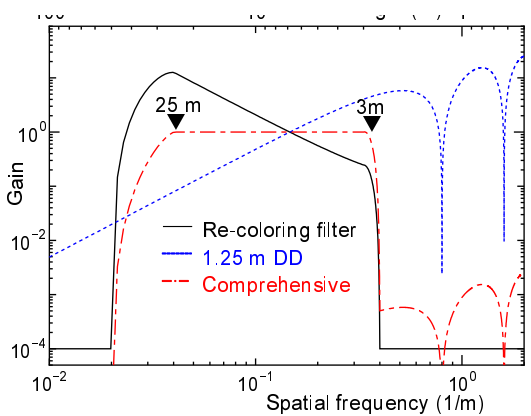

(b) Frequency response functions

Figure 12: Re-coloring filter (from DD signal to D1).

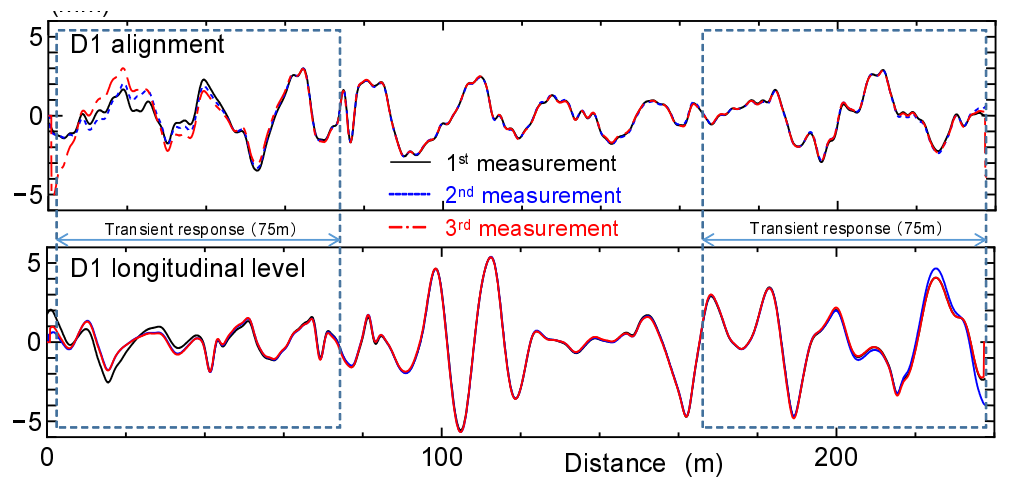

Figure 13: Repeatability of D1 track geometry calculated based on signals measured from LRSX01. 
(a) At all data points except for the transient response section, the difference between the maximum and minimum values of the three measurements is calculated.

(b) A histogram of the repeatability error is created to find the maximum error.

A histogram of the repeatability error for D1 alignment and D1 longitudinal level are shown in Figure 14. Maximum error of the D1 alignment was $0.174 \mathrm{~mm}$, and that of the longitudinal level was $0.197 \mathrm{~mm}$. It was confirmed that this prototype sufficiently satisfies an uncertainty of $\pm 1 \mathrm{~mm}$ as required by EN138484.
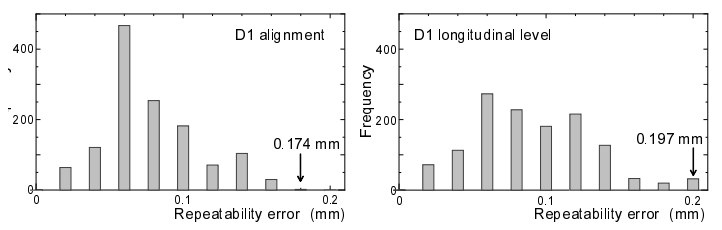

Figure 14: Repeatability error for D1 track geometry (Difference in three measurements at all measurement points).

\section{Conclusions and future remarks}

The following conclusions were drawn from our development:

(a) A brand-new track measuring principle named "differential difference method (DD-method)" based on a modified two-point angle method, which is used for measuring precise shapes, was developed for track geometry measurement.

(b) Using this revolutionary DD-method, track irregularity, which does not depend on the measurement velocity, can be obtained by a simple processing.

(c) Differential difference data can be completely converted to versine by simple and stable moving sum operation.

(d) A prototype trolley having a two-part structure and called LRSX01 was successfully developed based on the DD-method. It can be divided into two beams and is very lightweight at $9.5 \mathrm{~kg}$.

(e) Repeatability tests with the LRSX01 confirmed that the measurement error is very small in a wide wavelength range.

(f) A conversion filter was designed for track irregularity index "D1" as defined by European standards (EN13848). Repeatability error of the D1 satisfied the EN standard.

Further development and improvements are as follows.

(a) Development of PC display software

(b) Addition of a switch geometry measurement function 
(c) Complete conformance with EN13848-4

A commercial model of LRSX01 is expected to go on sale globally in April 2017.

\section{References}

[1] Yasukuni Naganuma, Mamoru Tanaka, and Kimihiro Ichikawa. High-speed track inspection car in the new dr. yellow. In Proc. of the World Congress on Railway Research (WCRR 2001), 2001.

[2] Yasukuni Naganuma, Seiji Fukushima, and Masaru Morimoto. Truly portable track recording equipment and its application for track quality management. In Proc. of the World Congress on Railway Research (WCRR 2003), pages 1313-1323, 2003.

[3] Coenraad Esveld. Modern Railway Track. MRT-Productions, 1989.

[4] S Kiyono, T Kume, Y Asakawa, and O Kamada. Differential laser autocollimation method ( $2^{\text {nd }}$ report) (in japanese). Journal of JSPE, 57(4): 652, 1991.

[5] Yasukuni Naganuma, Mikihito Kobayashi, and Takayuki Okumura. Inertial measurement processing techniques for track condition monitoring on shinkansen commercial trains. Journal of Mechanical Systems for Transportation and Logistics, 3(1): 315-325, 2010. 